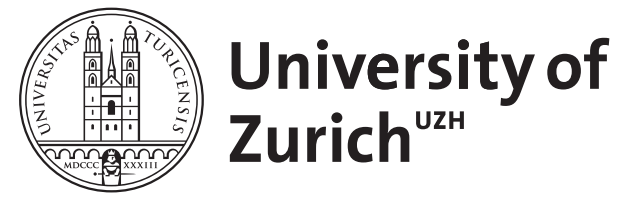

\title{
Wechseljahre und ihre Störungen aus ayurvedischer Sicht
}

Kneip, B

DOI: https://doi.org/10.1159/000342678

Posted at the Zurich Open Repository and Archive, University of Zurich ZORA URL: https://doi.org/10.5167/uzh-66593

Journal Article

Published Version

Originally published at:

Kneip, B (2012). Wechseljahre und ihre Störungen aus ayurvedischer Sicht. Schweizerische Zeitschrift für Ganzheitsmedizin / Swiss Journal of Integrative Medicine, 24:307-312.

DOI: https://doi.org/10.1159/000342678 


\section{Wechseljahre und ihre Störungen aus ayurvedischer Sicht}

\author{
Bettina Kneip \\ Institut für Naturheilkunde, UniversitätsSpital Zürich, Schweiz
}

\section{Schlüsselwörter}

Ayurvedische Medizin - Wechseljahre - Postmenopause .

Ayurvedische Phytotherapie - Phytotherapie - Kräutertherapie

\section{Zusammenfassung}

Dieser Artikel soll einen Überblick über die Möglichkeiten und Grenzen der traditionellen indischen Medizin (TIM) - ayurvedische Medizin - zur Behandlung der häufigsten Symptome bei Wechseljahrbeschwerden geben. Einleitend wird ein kurzer Überblick über die historische Entwicklung der ayurvedischen Medizin gegeben. Es folgt ein Auszug über ausgewählte Grundprinzipien der ayurvedischen Medizin, das Zusammenspiel der Grundelemente Luft, Raum, Feuer, Erde und Wasser und deren Zusammenhang in der Entstehung von Krankheiten, wobei Wechseljahrbeschwerden im Vordergrund stehen. Anschliessend werden sowohl Empfehlungen zur Ernährung als auch therapeutische Ansätze für pflanzliche Zubereitungen, Aromaöle, Massage, Sport und Entspannungsverfahren beschrieben.

\section{Keywords}

Ayurvedic medicine - Menopause - Postmenopause .

Ayurvedic phytotherapy $\cdot$ Phytotherapy $\cdot$ Herbal medicine

\section{Summary}

Menopause and Its Disorders from the Ayurvedic Point of View

This article gives an overview of the possibilities and limitations of traditional Indian medicine (TIM) - Ayurvedic medicine - for the treatment of the most common symptoms of the menopause. It provides a brief historical overview of the development of Ayurvedic medicine, followed by a description of selected basic principles. The interaction of the basic elements air, space, fire, earth and water is presented in regard to the development of diseases and disease symptoms, in particular menopausal symptoms. Finally, recommendations for diet therapeutic approaches regarding herbal medicine, aromatherapy oils, massage, sports and relaxation techniques are given.

\author{
Mots-clés \\ Médecine ayurvédique - Ménopause · Post-ménopause . \\ Phytothérapie ayurvédique · Phytothérapie - Traitement \\ par les plantes aromatiques
}

\section{Résumé}

Ménopause et troubles associés dans la médecine ayurvédique L'objectif de cet article est de donner un aperçu des possibilités et limites de la médecine indienne traditionnelle (MIT), ou médecine ayurvédique, dans le traitement des symptômes les plus courants de la ménopause. Nous reviendrons tout d'abord rapidement sur le développement de la médecine ayurvédique d'un point de vue historique. Nous nous pencherons ensuite sur certains principes clés de la médecine ayurvédique, par exemple le rôle des éléments fondamentaux (l'air, l'éther, le feu, la terre et l'eau) ainsi que leur lien avec l'apparition de pathologies fréquement associées à la ménopause. Enfin, nous émettrons des recommandations sur l'alimentation mais aussi les applications thérapeutiques des préparations à base de plantes ainsi que sur les huiles aromatiques, les massages, le sport et les techniques de relaxation.

\section{Einleitung}

Die ayurvedische oder traditionelle indische Medizin (TIM) kann als eines der ältesten Medizinsysteme sinnvolle therapeutische Anregungen und Erweiterungen zu unserem heutigen medizinischen Menschenbild beisteuern. Neben der Heilung und Prävention von Krankheiten finden wir hier ein umfangreiches, grundlegendes medizinisches Wissen, das den Menschen, genau wie in der traditionell europäischen Naturheilkunde (TEN), als ganzes Individuum betrachtet - Geist, Körper, Seele -, was sich bereits in dem Sanskritwort Ayurveda (d.h. Wissen oder Wissenschaft vom Leben) ausdrückt. Die Veden beinhalten den ältesten Teil der schriftlichen Aufzeichnungen der indischen Literatur [1]. Der Rik-Veda ist der Grundstein und der älteste Teil der Veden; im Artharva-Veda sind

\section{KARGER}

Fax +497614520714 Information@Karger.de www.karger.com (c) 2012 S. Karger GmbH, Freibur 1015-0684/12/0245-0307\$38.00/0
Dr. med. Bettina Kneip

Institut für Naturheilkunde

UniversitätsSpital Zürich

Sonneggstrasse 6, 8006 Zürich, Schweiz

Tel, +41 442552460 Bettina.Kneip@usz.ch 
viele Bezugspunkte in Form von metrischen Gesängen niedergelegt, die auch heutzutage noch in Indien von Pandits (indischen Gelehrten) rezitiert werden und mit den Grundstein der vedischen Medizin bilden [1]. Die traditionsreichen Gesänge oder Heilgesänge wurden 2003 in die UNESCO-Liste der Meisterwerke des mündlichen und immateriellen Erbes aufgenommen (UNESCO: Presentation of the Masterpieces Proclaimed 2003).

Vergleichbare Prinzipien, die sich teilweise und viele Jahre später aus den ayurvedischen Schriften entwickelt haben, finden sich in fast allen Medizinalsystemen wieder, da sie sich mit ihrer Elementenlehre als sehr grundlegend darstellen. Wir finden Ansätze unter anderem im Schamanismus, im Hinduismus, im Buddhismus, in der Chinesischen Medizin, in der hellenistischen Medizin und in der europäischen Naturmedizin. Die überlieferten klassischen Bücher, wie der Caraka-Samhita (Samhita bedeutet Sammelwerk), Sushruta-Samhita und Vagbhata-Samhita, bilden heute noch die Grundlage des Wissens an den indischen Universitäten [2]. Bereits in diesen klassischen, teilweise bis zu 1500 Jahren alten Lehrbüchern finden wir das ausführliche Wissen über Pathogenese, Kräutertherapie, Entgiftungstherapien, Biorhythmus, Pulsdiagnostik, Chirurgie, innere Medizin, Pädiatrie, Gynäkologie, Ernährungstherapie, Präventionsmedizin und Verjüngungsmedizin (Rasayana-Therapie) [2].

\section{Ausgewählte Grundprinzipien der ayurvedischen Medizin}

Der Leitgedanke der ayurvedischen Medizin findet sich zusammenfassend in seiner Definition über Gesundheit. Aus ayurvedischer Sicht ist Gesundheit nicht nur die Abwesenheit von Krankheit, sondern bedeutet das Gleichgewicht zwischen Körper, Geist und Bewusstsein:

Gesundheit besteht in einem ausgewogenen Zustand der Vata (Luft-Raum-Element), Pitta (Feuer-Wasser-Element) und Kapha (Erde-Wasser), der Gewebe (Dhatus), der Ausscheidungsprodukte (Malas) und der Verdauungssäfte (Agni) in Verbindung mit Klarheit bzw. Ausgewogenheit der Sinne, des Geistes und der Seele [3].

Dabei ist die ayurvedische Medizin ausgerichtet auf die elementaren Prinzipien von Existenz - die Tridoshalehre: Luft, Raum, Feuer, Erde und Wasser. Jegliche Daseinsform und Materie, in denen wir die unterschiedlichen Qualitäten der Elemente ausgedrückt finden, lassen sich auf diese Grundelemente zurückführen. Dies trifft sowohl auf den menschlichen Körper und Geist zu, mit all seinen verschiedenen Organsystemen und Funktionen, als auch auf die äusseren Einflüsse, die auf unseren Organismus Einfluss nehmen, wie Jahreszeiten, Tageszeiten, Lebensdekaden und all die Dinge, die wir täglich aufnehmen: Nahrungsmittel, Eindrücke, Gefühle, Stimmungen usw.
Der Körper ist durch die permanenten Einflüsse bestrebt, die individuelle Homöostase bzw. das individuelle Gleichgewicht der Doshas zu erhalten oder wiederherzustellen [1], d.h. die Grundelemente Vata, Pitta und Kapha in den individuellen Konstitutionszustand zurückzuführen. So ist der Mensch ausgeglichen und fühlt sich gesund. Ungleichgewichte können anhand von Befindlichkeitsstörungen spürbar sein, wobei diese häufig noch nicht mit den herkömmlichen medizinischen apparativen Analysetechniken messbar sind. Bei Fortbestehen des Störungsbildes kommt es früher oder später zu manifesten Krankheitsbildern sowie im weiteren Verlauf möglicherweise zur Chronifizierung.

Eine ausführliche, umfassende Krankheitserhebung beinhaltet auch die Berücksichtigung des Geistes, der Psyche und der Seele. Wenn wir immer wieder falsche Denkmuster und Vorstellungen übernehmen, entwickeln wir in unserem Körper eine entsprechende Biochemie oder Psychosomatik - Gesichtspunkte, die auch von der modernen Neuroimmunologie aufgegriffen werden [4]. Der Geist ist über afferente und efferente Bahnen mit allen Zellen des Körpers verbunden und kann darüber Antworten produzieren und erhalten - teilweise bewusst, teilweise unbewusst [5]. Deepak Chopra beschreibt es in seinem E-Book «Magical Mind, Magical Body» folgendermassen: «Der Körper ist eine Projektion des Bewusstseins.» Es scheint möglich, dass die Wissenschaft der Neuroimmunologie dort angekommen ist, wo die Intuition seit jeher bestand.

Entspannungstechniken finden sich im Yoga beschrieben (unterschiedliche Meditationsarten), einer Schwesterdisziplin des Ayurveda $[1,6]$. Mit diesen Techniken können wir Voraussetzungen schaffen, die eine Basis für eine psychische oder seelische Ausgeglichenheit im Hinblick auf eine Umstellung der Denkmuster, auch im Sinne einer Bewusstwerdung, ermöglichen; nicht unwesentlich dabei ist die Schulung des inneren Beobachters.

\section{Die Lebensdekaden aus ayurvedischer Sicht}

Den ayurvedischen Vorstellungen entsprechend kann man das menschliche Leben in drei Dekaden einteilen [6]:

- Die Zeit vom Säugling bis zur Pubertät wird vom Kapha-Element (Erde und Wasser) dominiert. Folgende Eigenschaften stehen in dieser Lebensdekade im Vordergrund: Wachstum, Entwicklung, Aufbau des Immunsystems und der Abwehr, Stabilität finden in den Veränderungen des Lebens, grösseres Schlafbedürfnis. Typische Krankheitserscheinungen sind Infektionskrankheiten (Kinderkrankheiten), Erkältungskrankheiten, Adenoide, Wachstumsstörungen usw. [3]. 
- Nach der Kapha-Zeit folgt die Pitta(Feuer)-Lebensphase. In dieser Lebensdekade geht es um Themen wie Leben bestreiten, Familie ernähren und Geld verdienen. Wir benötigen also unsere gesamte Lebensenergie, das «Feuer», für diesen Zeitraum. Typische Pitta-Krankheiten sind Magenulzera, Cholezystolithiasis (Pitta-Kapha-Störung), Inflammationen aller Art und Hitzestörungen [3].

- In der dritten Lebensdekade überwiegt das Luft-RaumElement, das Vata. Jetzt kommt die Zeit des Loslassens, der Lebensweisheit, der Reduktion auf das Wesentliche. Typische Vata-Krankheiten sind Schlafstörungen, Depression, Prostataleiden, Unruhezustände und Trockenheitszustände (Haut, Schleimhäute) [3].

\section{Wechseljahre im Lebenszyklus der Frau}

Die Wechseljahre umschreiben einen Wandel im Leben einer Frau (und des Mannes) zwischen der Feuer(Pitta)und Luft(Vata)-Lebensdekade. Die Übergänge zwischen den Lebensabschnitten sind für den Organismus eine anspruchsvolle Zeit, denn je mehr sich der Körper in einem Ungleichgewicht befindet, umso schwieriger gestalten sich die Anpassungsprozesse. Im Zusammenhang mit den Wechseljahren (der erste Lebensabschnitt schliesst mit der Pubertät ab, der zweite mit den Wechseljahren) kann das ein Ungleichgewicht von Vata, Pitta und Kapha bedeuten, woraus folgende Symptome/Beschwerden und ihre Doshazuordnungen resultieren können:

- Hitzewallungen, Gereiztheit, Ungeduld, Wut, Ärger und Entzündungen als Pitta-Störungen (Abb. 1);

- Schlafstörungen, Unruhezustände, Angststörungen, Depressionen, Nervenleiden und vaginale Trockenheit als Vata-Störungen (Abb. 2);

- Trägheit, Gewichtszunahme, Antriebsschwäche und Kopfschmerzen als Kapha-Störungen (Abb. 3).

Man kann den Körper nach den ayurvedischen Prinzipien mit der adäquaten Ernährungs- und Lebensweise sowie mit Kräuter- und Aromatherapien unterstützen, sodass die Übergänge fliessender ausfallen.

\section{Ernährung und Wechseljahre}

Mit den vielfältigen Nahrungsmitteln können wir einen nicht unwesentlichen Einfluss auf unsere körperliche Befindlichkeit und auch auf Krankheiten nehmen, da jedes Nahrungsmittel das körperliche Gleichgewicht in seinem Urcharakter (Vata, Pitta, Kapha) beeinflussen kann. So ist es möglich, dass Symptome mit den einzelnen Nahrungsmitteln verstärkt oder bei entsprechender Rücksichtnahme auf die Doshas gelindert werden können.

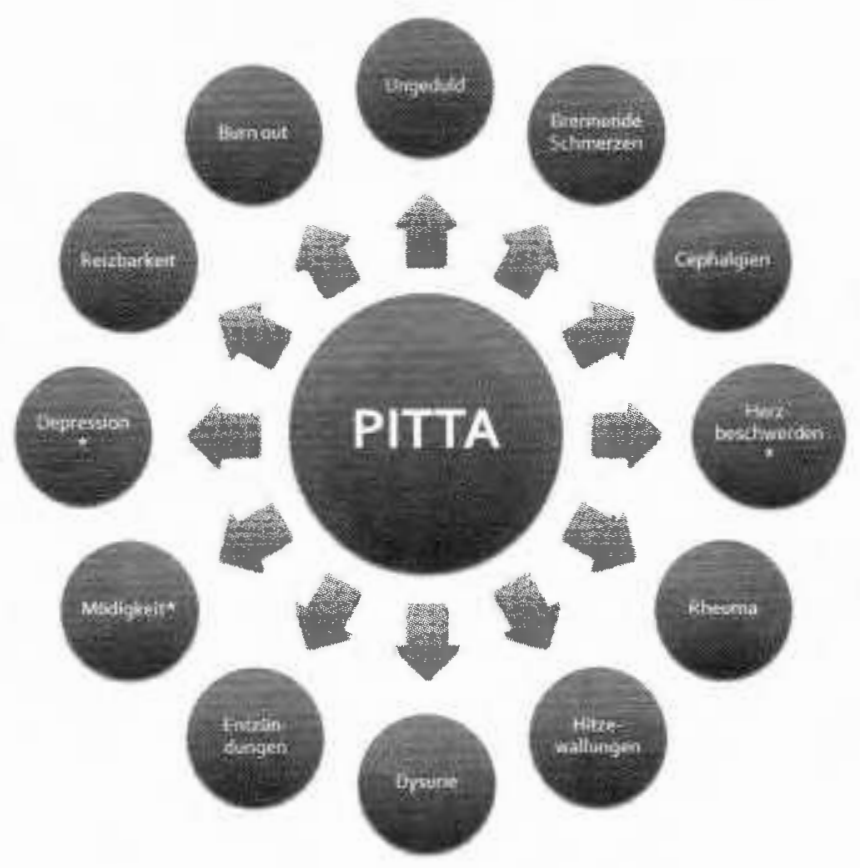

Abb. 1. Wechseljahrprobleme ausgelöst durch ein Pitta-Ungleichgewicht ( ${ }^{*}$ Symptomkomplexe, die auch durch Vata oder Kapha verursacht sein können).

\section{Hitzewallungen}

Bei Hitzewallungen wird empfohlen, folgende Nahrungsmittel zu reduzieren (d.h. sie verstärken die innere Hitze) [7]:

- Alkohol,

- Chili,

- rote Gemüse inklusive Tomaten,

- Ingwer in grossen Mengen,

- Kaffee,

- Schwarztee,

- Süssigkeiten mit hohem Zuckeranteil,

- saures Obst,

- rotes Fleisch,

- saurer Joghurt,

- stark gesalzene Kost,

- Lachs,

- Roggen.

Folgende Nahrungsmittel können Hitze positiv beeinflussen, d.h. lindern [7]:

- grünes Gemüse, Fenchel,

- Bitterstoffe (Gelbwurz, Chicorée, Artischocken),

- gelbe Linsen,

- alle süssen Obstsorten,

- Mineralien wie Kalzium, Magnesium und Zink,

- Milch, Milchprodukte,

- Getreidearten (Ausnahme: Roggen). 


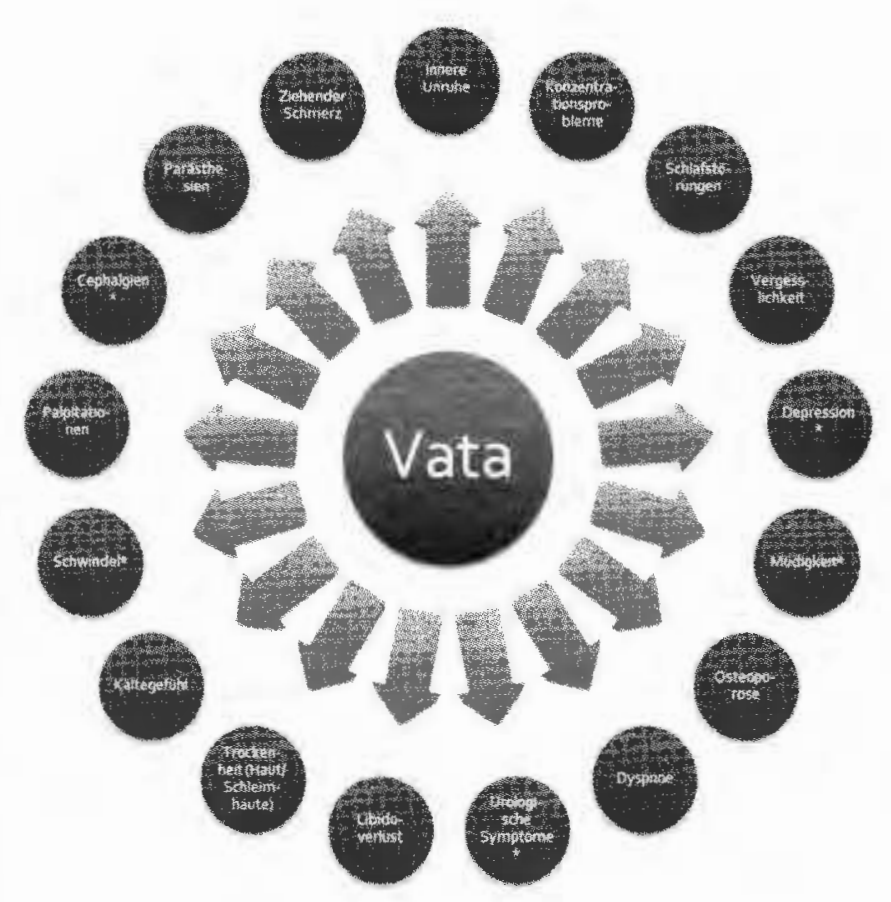

Abb. 2. Wechseljahrprobleme ausgelöst durch ein Vata-Ungleichgewicht $\left({ }^{\star}\right.$ Symptomkomplexe, die auch durch Pitta oder Kapha verursacht sein können).

Unruhe, Schlafstörungen und Trockenheit

Folgende Nahrungsmittel sollten bei Unruhe, Schlafstörungen und Trockenheit (Haut, Schleimhäute, Augen) gemieden werden [7]:

- Salat,

- Rohkost,

- kalte Speisen und Getränke,

-Glace,

- Kohlarten,

- Kaffee,

- Weisswein,

- Schwarztee.

Folgende Nahrungsmittel können Unruhe, Schlafstörungen und Trockenheit (Haut, Schleimhäute, Augen) positiv beeinflussen [7]:

- warme, frisch gekochte Speisen,

- Porridge zum Frühstück,

- warme Suppen zum Nachtessen,

- gut gewürzte Speisen mit Ingwer, Koriander, Gelbwurz, Kümmel, Kardamom, Zimt, Vanille,

- Süssspeisen wie Milchreis oder Griessbrei,

- Fisch,

- Geflügel.

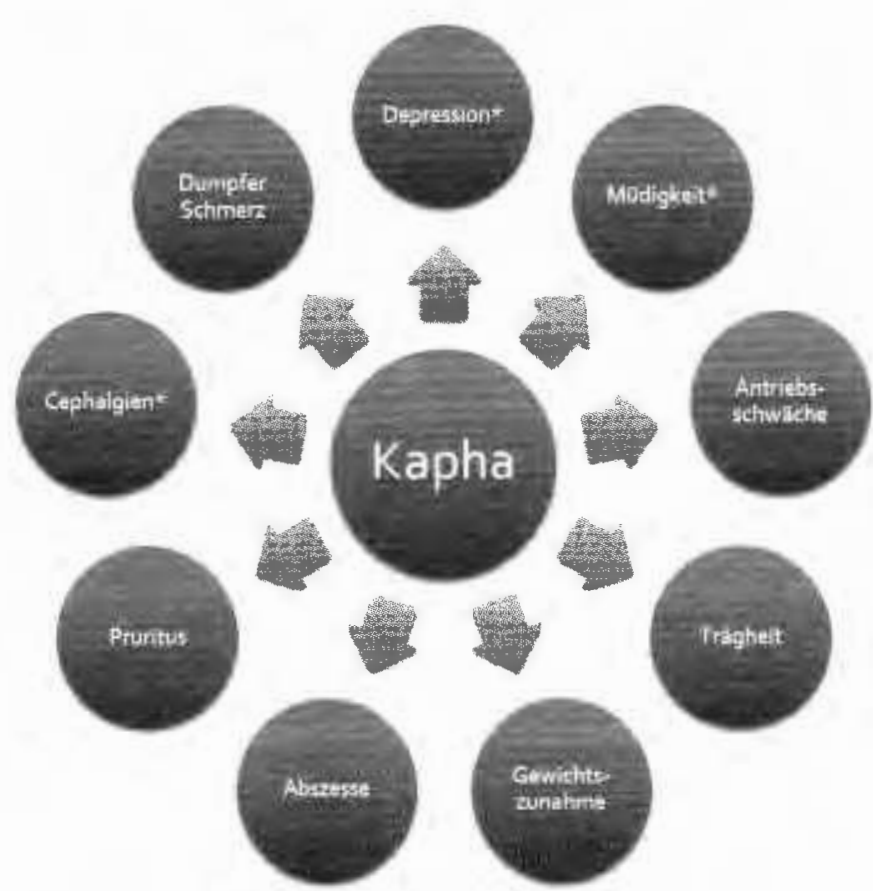

Abb. 3. Wechseljahrprobleme ausgelöst durch ein Kapha-Ungleichgewicht $\left({ }^{*}\right.$ Symptomkomplexe, die auch durch Pitta oder Vata verursacht sein können).

Übergewicht, Müdigkeit, Trägheit und Antriebsschwäche Folgende Nahrungsmittel können Übergewicht, Müdigkeit, Trägheit und Antriebsschwäche verschlimmern [7]: - frittierte, ölige, fettige Speisen,

- Milch, Milchprodukte,

- Süssigkeiten.

Folgende Nahrungsmittel können Übergewicht, Müdigkeit, Trägheit und Antriebsschwäche verbessern:

- Rohkost,

- Gemüse,

- trockene, herbe Früchte, Beerenobst,

- Hülsenfrüchte,

- scharfe, erwärmende Gewürze,

- Gerste, Hirse, Buchweizen,

- warme Getränke.

Generell sollte auf eine frische, abwechslungsreiche Ernährung mit viel Obst und Gemüse geachtet werden, da deren sekundäre Pflanzenstoffe wissenschaftlich nachgewiesene [8], positive Auswirkungen auf unsere Zellen haben (z.B. bei Krebswachstum, Entzündungen). 


\section{Getränke und Wechseljahre}

Bei Hitzewallungen hilft 3- bis 5-mal täglich Rosenwasser trinken [6]. Rosenwasser erhält man in der Apotheke, und es reichen je nach Geschmacksempfinden 1-2 Teelöffel Rosenwasser pro Glas Wasser.

Auch folgende Teemischung hat sich bewährt: Je ein halber Teelöffel Korianderpulver, Kreuzkümmelpulver, Kurkuma und Fenchelsamen mit einem halben Liter heissem Wasser überbrühen, 5-10 min ziehen lassen und tassenweise tagsüber trinken [9].

Bei Stimmungsschwankungen wird in der ayurvedischen Medizin Süssholz eingesetzt. 1 Esslöffel getrocknete und zerkleinerte Wurzel (in der Apotheke oder Drogerie erhältlich) mit 1 Liter kaltem Wasser erhitzen, 10 min kochen lassen und dann absieben. Über den Tag verteilt trinken. Cave: Süssholztee kann hohen Blutdruck und Elektrolytstörungen verursachen [10].

Kühlend auf den Organismus wirkt sich auch AloeVera-Frischpflanzensaft aus. Man kann ihn in Drogerien beziehen und nimmt täglich 1-3 Teelöffel Aloe-Vera-Saft über den Tag verteilt ein. Dies kann auch bei bestehender Obstipation (Vata-Störung) hilfreich sein [3].

Alternativ gegen Hitzewallungen eignet sich auch Granatapfelsaft mit einem Teelöffel Rohrohrzucker oder Zitronengrastee. Man kann dieses Getränk, falls nötig, 2- bis 3-mal täglich einnehmen [6].

\section{Ayurvedische Phytotherapie und Wechseljahre}

Auch durch die Phytotherapie kann Einfluss auf die Doshas in unserem Körper genommen werden.

Pflanzliche Zubereitungen, die Vata reduzieren [10]:

- Bacopa monnieri (Nabelkraut): abends einen Teelöffel vom Saft der Blätter;

- Withania somnifera (Winterkirsche): 2,5 g Pulver der Wurzel mit etwas Milch einnehmen;

- Emblica officinalis (Aschfarbene Myrobalane): Aufguss der Samen (1 Teelöffel auf 1 Tasse heisses Wasser);

- Commiphora myrrha (Echte Myrrhe): $200 \mathrm{mg}$ Pulver mit $200 \mathrm{ml}$ Wasser 3-mal täglich einnehmen;

- Aegle marmelos (Marmelos); 1 g pulverisierte Früchte mit $100 \mathrm{ml}$ Wasser 3-mal täglich;

- Glycyrrhiza glabra (Süssholz): $3 \mathrm{~g}$ Wurzel mit 1 Tasse heissem Wasser abkochen und 3-mal täglich trinken.

Pflanzliche Zubereitungen, die Pitta reduzieren:

- Saraca indica (Ashoka-Baum): 20 Tropfen eines konzentrierten Flüssigkeitsextrakts 3-mal täglich;

- Terminalia bellirica (Grüne belerische Myrobalane): Fruchtfleisch mit Salz, 3-mal täglich 1 Esslöffel einnehmen;
- Asparagus racemosus (Wilder Spargel): Abkochung der Wurzel (ca. $2 \mathrm{~cm}$ ) mit $250 \mathrm{ml}$ Milch (Rinde ist giftig!);

- Vitis vinifera (Echte Weinrebe): als Mischung mit G. glabra (8 Trauben mit 1 Teil G. glabra als kurze Abkochung in wenig Wasser) 3-mal täglich $30 \mathrm{ml}$ trinken;

- Emblica officinalis (Aschfarbene Myrobalane): Aufguss der Samen (1 Teelöffel auf 1 Tasse heisses Wasser);

- Terminalia arjuna (Arjuna Myrobalane): $20 \mathrm{ml}$ einer Abkochung der Rinde (1:10);

- Mimosa pudica (Schamhafte Sinnpflanze): Aufguss der Blätter (10 g in $250 \mathrm{ml}$ Wasser) 3-mal $10 \mathrm{ml}$ täglich trinken.

Pflanzliche Zubereitungen, die Kapha reduzieren:

- Commiphora mukul (Indische Myrrhe): älteres Harz als

Kräuterpresslinge 2-4 g täglich;

- Piper longum (Langer Pfeffer): gemahlenes, getrocknetes Pulver der Früchte 0,5-3 g täglich;

- Zingiber officinale (Ingwer): 0,5 Esslöffel Ingwersaft mit ein paar Tropfen Zitrone und Honig vor den Mahlzeiten einnehmen.

- Bei vaginaler Trockenheit hat sich ein Tampon getränkt mit Sesamöl als vaginale Therapie über Nacht bewährt [9]; alternativ: Vaginalzäpfchen mit Traubenkernöl und Granatapfel.

\section{Ayurvedische Massage}

Die ayurvedische Massage ist eine der wichtigsten unterstützenden Therapien im klassischen Ayurveda. Sie dient der Harmonisierung der Körperenergien; richtig angewendet, werden die Marma-Punkte (Energiepunkte, die weitgehend den Meridianpunkten der Chinesischen Medizin entsprechen) berücksichtigt und angeregt. Weiterhin leitet sie Stoffwechselgifte aus und unterstützt die tiefen Reinigungs- und Erneuerungsprozesse. Es werden neuroendokrine Impulse freigesetzt, die den Geist klären, das Nervensystem stärken, das Kanalsystem «Srotas» reinigen und dem Körper ein grosses Mass an vitaler Substanz verleihen [7, 9]. Dies ist z.B. hilfreich bei Übergewicht, Schlaflosigkeit, Verspannungen und Erschöpfung. Die Sehkraft wird verbessert, das Immunsystem und das allgemeine Abwehrsystem erfahren eine grundlegende Stärkung. Ölmassagen beruhigen das Nervensystem, regen den Lymphfluss an und bewirken eine glatte seidige Haut, was der Trockenheit im Alter entgegenwirkt [9].

Bei bestehenden Hitzeproblemen eignet sich Olivenöl von guter Qualität, bei Unruhe- und Schlafproblemen Lavendelöl. Man beginnt am Kopf, massiert leicht die Kopfhaut, die Ohrmuscheln (hier sitzen viele EnergieMarma-Punkte), den Hals und Nacken und die Brust. Diese wie eine Acht umkreisen. Dann wechselt man auf 
die Schultergelenke, diese umkreisen, die Oberarme in Richtung und Gegenrichtung streichen, die Ellbogengelenke umkreisen, die Unterarme ausstreichen, die Handund Fingergelenke umkreisen. Den Bauch im Uhrzeigersinn umkreisen, die Hüftgelenke umkreisen, Oberschenkel ausstreichen, Knie umkreisen, Unterschenkel ausstreichen, Knöchel und Füsse umkreisen. Das Öl anschliessend 10-15 min einziehen lassen, danach abduschen.

\section{Aromatherapie und Ayurveda}

Aromaöle werden aus Frischpflanzen oder Drogen durch Wasserdestillation gewonnen. [11]. Sie helfen mit ihren Inhaltsstoffen wie Säuren (mindern Vata und Pitta), Aldehyden (mindern Vata und Pitta), Monoterpenketonen, Sesquiterpenketonen, Diterpenketonen und Kumarinen, die bestehenden Symptome auszubalancieren. Bei Hitzestörungen helfen kühlende Öle wie Sandelholzöl oder Pitta-Aromaöle (Komposition aus kühlenden Aromaölen), bei Vata-Störungen Vata-Aromaöle, Lavendel- oder Orangenöl. Man kann die Fläschchen auch unterwegs mitnehmen und bei Bedarf die Aromen direkt inhalieren. Abends kann ein Taschentuch zur Inhalationstherapie eingesetzt werden (z.B. mit einem Vata-Aromaöl beträufeln und bei Schlafproblemen immer wieder an dem Taschentuch inhalieren).

\section{Sport und Ayurveda}

Regelmässige Sportübungen sind sowohl für die Knochen als Osteoporoseprophylaxe bei Kapha-Störungen in Form von Übergewicht als auch bei Hitze- und Schlafstörungen hilfreich. Idealerweise betreibt man täglich eine halbe Stunde Sport wie z.B. Nordic Walking oder Radfahren im Wechsel mit Aqua-Fitness. Alternativ kann man Yogaübungen für daheim, wie z.B. morgens den Sonnengruss
(Suryanamaskar) oder Yoga Asanas, ausführen. Es hält alle Gelenke beweglich und ist ein gutes Mittel, um den Tag positiv zu beginnen (Umstimmungstherapie) und die Elemente im Gleichgewicht zu halten [6].

\section{Verträglichkeit der ayurvedischen Medizin}

Es wird davon abgeraten, ayurvedische Medikamente ohne genaue Hersteller- und Produktionsangaben im Internet zu beziehen, da hier die Gefahr besteht, dass die Kräuter mit Pestiziden oder Schwermetallen belastet und nicht nach ursprünglichen ayurvedischen Rezepturen hergestellt worden sind. Zurzeit ist es noch recht schwierig, in der Schweiz ayurvedische Phytotherapeutika zu erhalten, die den westlichen Reinheitskriterien entsprechen. Oftmals ist es sinnvoll, auf importkontrollierte, toxinfreie chinesische Präparate zurückzugreifen, die in Schweizer Apotheken erhältlich sind.

\section{Grenzen der ayurvedischen Medizin}

Die ayurvedische Medizin ist bestens geeignet, Befindlichkeitsstörungen und chronische Erkrankungen zu behandeln sowie schulmedizinische Behandlungen bei schweren Krankheiten wie z.B. Krebs zu unterstützen. Bei neu aufgetretenen Symptomen, die sich ayurvedisch behandelt als therapieresistent erweisen oder länger bestehen, ohne bis anhin untersucht worden zu sein, sollte immer eine schulmedizinische Abklärung erfolgen, um schwerwiegende Krankheitsbilder nicht zu verpassen.

\section{Disclosure Statement}

Die Autorin erklärt, dass sie keinerlei Interessenkonflikte im Zusammenhang mit diesem Beitrag hat.

\section{Literatur}

1 Ranade S: Ayurveda-Wesen und Methodik. Heidelberg, Haug, 1994.

2 Porter R: Die Kunst des Heilens. Heidelberg, Spektrum, 1997.

3 Lad V: Selbstheilung mit Ayurveda. Baar, O.W. Barth, 1998.

4 Besedovsky HO, Rey AD: Physiology of psychoneuroimmunology: a personal view. Brain Behav Immun 2007;21:34-44.
5 Kasture HS: Concept of Ayurveda for Perfect Health and Longevity. Nagpur, Shree Baidyanath Ayurveda Bhavan, 1990.

6 Ranade S, Hosius C, Heckmann J: Ayurveda, Basislehrbuch. München, Urban \& Fischer, 2003.

7 Mathew S, Schmidauer G: Ayurveda-Medizin. Wien, Verlagshaus der Ärzte, 2010.

8 Watzl B, Leitzmann C: Bioaktive Substanzen in pflanzlichen Lebensmitteln. Stuttgart, Hippokrates, 1999.
9 Atreya: Ayurvedic Healing for Women. Delhi, Motilal Banarsidass, 2004.

10 Zoller A, Nordwig H: Heilpflanzen der Ayurvedischen Medizin. Heidelberg, Haug, 1997.

11 Saller R: Beiträge zur Phytotherapie. Phytotherapie und therapeutisch orientierte Forschung. München, Hans Marseille, 1993. 